\title{
Sequence analysis of Drd2, Drd4, and Dat I in SHR and WKY rat
} strains

\author{
Jonathan Mill12, Terje Sagvolden ${ }^{3}$ and Philip Asherson*1
}

Address: ${ }^{1}$ MRC SGDP Centre, Institute of Psychiatry, London, UK, ${ }^{2}$ Cell and Molecular Biology Division, Toronto Western Research Institute, University Health Network, Toronto, ON, Canada and ${ }^{3}$ University of Oslo, Oslo, Norway

Email: Jonathan Mill - j.mill@iop.kcl.ac.uk; Terje Sagvolden - terje.sagvolden@medisin.uio.no; Philip Asherson* - p.asherson@iop.kcl.ac.uk

* Corresponding author

Published: 15 December 2005

Behavioral and Brain Functions 2005, I:24 doi:10.1 186/I744-908I-I-24
Received: 23 August 2005

Accepted: 15 December 2005

This article is available from: http://www.behavioralandbrainfunctions.com/content/l/I/24

(C) 2005 Mill et al; licensee BioMed Central Ltd.

This is an Open Access article distributed under the terms of the Creative Commons Attribution License (http://creativecommons.org/licenses/by/2.0), which permits unrestricted use, distribution, and reproduction in any medium, provided the original work is properly cited.

\begin{abstract}
Background: The Spontaneously Hypertensive Rat (SHR) shows a number of behaviours that closely parallel those seen in children with attention-deficit hyperactivity disorder. These include motor hyperactivity, excessive responses under a fixed-interval/extinction schedule, difficulty in acquiring operant tasks and increased sensitivity to immediate behavioural reinforcement. As in children with ADHD, the behavioural and cognitive deficits in the SHR are responsive to stimulants, including $d$-amphetamine and d,l-methylphenidate. The non-hyperactive Wistar Kyoto (WKY) rat strain is often used as a control in behavioural studies of the SHR, and WKY itself has been suggested to be a useful animal model of depression. Numerous studies have shown that dopaminergic neurotransmission is altered between the two strains. Human genetic studies have found associations between several dopaminergic genes and both ADHD and depression.
\end{abstract}

Methods: We sequenced three candidate dopaminergic genes (Drd2, Drd4, and Dat I) in the SHR and WKY to identify between-strain sequence differences.

Results: No between-strain sequence differences were found in either Drd2 or Drd4, but several variations were found in the Dat I gene that encodes the dopamine transporter.

Conclusion: It is plausible that DNA sequence changes in the Dat I gene account for some of the behavioural differences observed between the SHR and WKY strains. Future work will focus on elucidating the functional effects of the observed polymorphisms.

\section{Background}

Attention-deficit hyperactivity disorder (ADHD) is a common neurobehavioural disorder defined by symptoms of developmentally inappropriate inattention, impulsivity and hyperactivity. It is estimated that between $3-6 \%$ of school age children are diagnosed with ADHD, making it the most prevalent disorder of childhood. While the precise aetiology of ADHD is yet to be ascertained, it is clear from numerous family, twin and adoption studies that genetic factors play in a key role in susceptibility to the disorder. Polymorphisms in several genes have been associated with ADHD, with a particular focus on genes implicated in monoamine neurotransmission [1].

A number of animal models have been proposed for ADHD and these have helped inform research into the biological basis of the clinical disorder. The spontaneously hypertensive rat (SHR) is one of the most widely 
Table I: The chromosomal location, amino-acid length and human-rat homology for the three genes sequenced in this study.

\begin{tabular}{cccccc}
\hline $\begin{array}{c}\text { Gene } \\
\text { ID }\end{array}$ & Location & Human & AA & Rat & Homology \\
$(\%)$
\end{tabular}

validated animal models of ADHD [2,3]. The SHR shows a number of behaviours that closely parallel those seen in children with ADHD including motor hyperactivity, increased impulsiveness and deficient sustained attention $[3,4]$. Furthermore, like children with ADHD, the SHR is more sensitive to immediate behavioural reinforcement and less sensitive to delayed reinforcement than nonhypertensive WKY control rats [3,4]. The behavioural and cognitive deficits in the SHR are responsive to stimulants, including d-amphetamine and d,l-methylphenidate [5]. Finally, several studies have shown that dopaminergic and noradrenergic neurotransmission is altered in the SHR compared to the WKY, strongly implicating these systems in the aetiology of ADHD [2,6,7].

The WKY strain, from which the SHR was initially derived by selective outbreeding [8], is itself proposed to be a model of another psychiatric condition - depression. As for ADHD, the aetiology of depression has been shown to be strongly influenced by genetic factors [9] and dysregulation of the dopaminergic system has been strongly implicated [10]. WKY rats have been shown to exhibit exaggerated neuroendocrine and behavioral responses to stress that exceed normal controls and are especially prone to develop stress-induced depressive disorder $[11,12]$. A recent study by Will et al found that selectively bred WKY rats were a particularly good animal model of depression and hyper-responsiveness to anti-depressants [13]. Interestingly, the dopamine neurotransmitter pathway has been strongly implicated in the depression-like behaviours exhibited by WKY rats. Jiao et al observed differences in the density and distribution of dopamine transporter sites in WKY rats that may lead to altered modulation of synaptic dopamine levels in the cell body and mesolimbic regions [14].

In this study we have sequenced three dopaminergic candidate genes (Drd2, Drd4, and Dat1) in the SHR and WKY rat strains to identify potential genetic variants that may explain some of the behavioural differences observed between the two strains.

\section{Methods}

Blood was obtained from animals housed at the University of Oslo and DNA was extracted using a standard protocol [15]. Bioinformatic analyses were performed to identify regions in the rat genome containing homologues of human ADHD candidate genes using sequence data deposited in the Rat Genome Database http:// rgd.mcw.edu/. Where no annotated rat genome sequence was available, BLAST searches were performed on raw sequence data to identify the relevant regions http:// www.ncbi.nlm.nih.gov/BLAST/. Primers were designed to span the promoter and exonic regions of three candidate genes (Drd2, Drd4, and Dat1) using Primer Express software (Applied Biosystems, Foster City, CA, USA). To conserve space oligo sequences are not given in this manuscript, but are available from the authors on request. The chromosomal location, amino-acid length and human-rat homology for the three genes sequenced in this study can be seen in Table 1 .

The exonic regions were amplified on an MJ PTC-225 thermal cycler (MJ Research) with an initial 9-min denaturing step at $95^{\circ} \mathrm{C}$, followed by 35 cycles of $93^{\circ} \mathrm{C}$ for 1 $\min , 55^{\circ} \mathrm{C}$ for $1 \mathrm{~min}$ and $72^{\circ} \mathrm{C}$ for $1 \mathrm{~min}$, and a final extension phase of $72^{\circ} \mathrm{C}$ for $10 \mathrm{~min}$. Reactions were performed in $22 \mathrm{ul}$ volumes and included $50 \mathrm{ng}$ of genomic DNA, $1.5 \mathrm{mM} \mathrm{MgCl} 2,0.2 \mathrm{mM}$ dNTP's, $10 \mathrm{mM}$ GeneAmp $10 \times$ PCR Gold Buffer (PE Applied Biosystems, Foster City, US) and 1 unit of AmpliTaq Gold (PE Applied Biosystems, Foster City, US). PCR products were run out on a $2 \%$ agarose gel stained with ethidium bromide and analysed under UV light. PCR products were purified using Qiagen Gel Extraction Columns (Qiagen, Crawley, UK). Following purification, forward and reverse dye terminator sequencing was carried out using ABI BigDye V3.0 and samples run on either an ABI 377 or 3100 machine (Applied Biosystems, Foster City, CA, USA). Sequencing traces were analysed using Sequencher software (GeneCodes Corporation, Ann Arbor, MI, USA), and multiple sequences aligned to aid mutation detection.

\section{Results \\ Drd2}

The rat $\operatorname{Drd} 2$ gene is located on chromosome $8 \mathrm{q} 23$ and is $95 \%$ homologous with the human DRD2 gene. The putative promoter and exonic regions of Drd2 were sequenced. No differences were observed between the SHR and WKY strains, and both sequences were identical to those in the Rat Genome Database http://rgd.mcw.edu/ 
Table 2: Variants noted in the Drd4 gene in both WKY and SHR strains compared to the sequence deposited in the Rat Genome Database http://rgd.mcw.edul.

\begin{tabular}{cc}
\hline Location & Variation \\
\hline Promoter region & GATGAA[G/T]AGTGAG* \\
Intron I & GGCGCG (not present in WKY/SHR) \\
Intron 2 & CACA (2 extra CA motifs in SHR/WKY) \\
Intron 2 & GAATGG[A/G]GACATA*
\end{tabular}

*WKY/SHR allele given second.

\section{Drd4}

The rat Drd4 gene is located on chromosome 1q41 and shares $75 \%$ homology with the human DRD4 gene. The putative promoter, exons, and introns of Drd4 were sequenced. No differences were found between the SHR and WKY strains, although both were found to contain non-coding sequence differences from the Brown Norway Rat sequence available in the Rat Genome Database (see Table 2). These differences included two single nucleotide polymorphisms (SNPs), one located in the putative 5' promoter region of the gene and the other in intron 2, along with length variation at a CA microsatellite repeat in intron 2. In addition, neither the WKY or SHR strains were found to have the short first intron (GGCGCG) present between exon 1 and 2 in the sequence available in the Rat Genome Database.

\section{Dat I}

The rat Dat1 gene is located on chromosome $1 \mathrm{p} 11$ and is 94\% homologous with the human DAT1 gene. The putative promoter and exonic regions of Dat1 were sequenced. The exon 3 amplicon could not initially be amplified by PCR in the WKY strain (see Figure 1a). Subsequent PCR amplification using more distal primers suggested the presence of extra sequence in the SHR compared to the WKY (see Figures $1 b$ and 1c). Sequencing of this region highlighted a synonymous single base change $(\mathrm{T} \rightarrow \mathrm{C})$ within the coding sequence of exon 3 (see Figure 2), and a $160 \mathrm{bp}$ section of sequence immediately upstream of exon 3 present in SHR but not WKY. Bioinformatic analysis of the public rat genome database demonstrated that this 160 bp sequence is also present $~ 1000$ bp upstream (in intron 4 of Dat1).

\section{Discussion}

In this study we sequenced three dopaminergic genes to examine differences between SHR and WKY rat strains. No between-strain sequence differences were found in genes encoding either the dopamine D2 receptor (Drd2) or the dopamine D4 receptor (Drd4), although for Drd4 both the WKY and SHR strains were found to differ from the sequence available in the Rat Genome Database http:// rgd.mcw.edu/. In contrast, several between-strain variations were found in the dopamine transporter gene (Dat1). Although none of the sequence changes results in a direct coding change to the DAT protein, it is plausible that they may alter expression-related processes such as transcription or splicing efficiency. Alternatively, it is possible that these changes are markers of other linked mutations carried on the same chromosome. These results are interesting given that the SHR and WKY strains are considered to be valid models of ADHD and depression respectively, and the postulated role of disrupted dopaminergic neurotransmission in both disorders.

It is pertinent that abnormalities in DAT expression and functioning have been noted in both rat strains. SHR

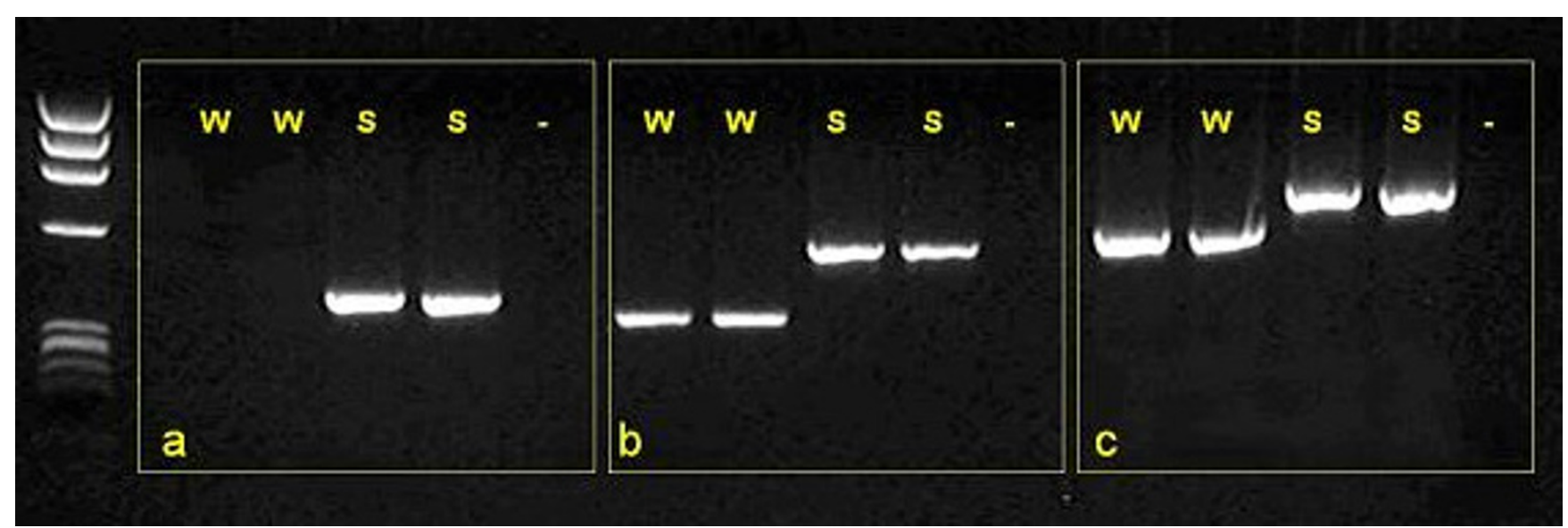

Figure I

PCR amplification of Dat l exon 3 using three alternative primer pairs on SHR (S) and WKY (W) DNA. On WKY DNA the reaction did not work using primer set (a) and resulted in a smaller PCR product than seen using SHR DNA with sets (b) and (c). 


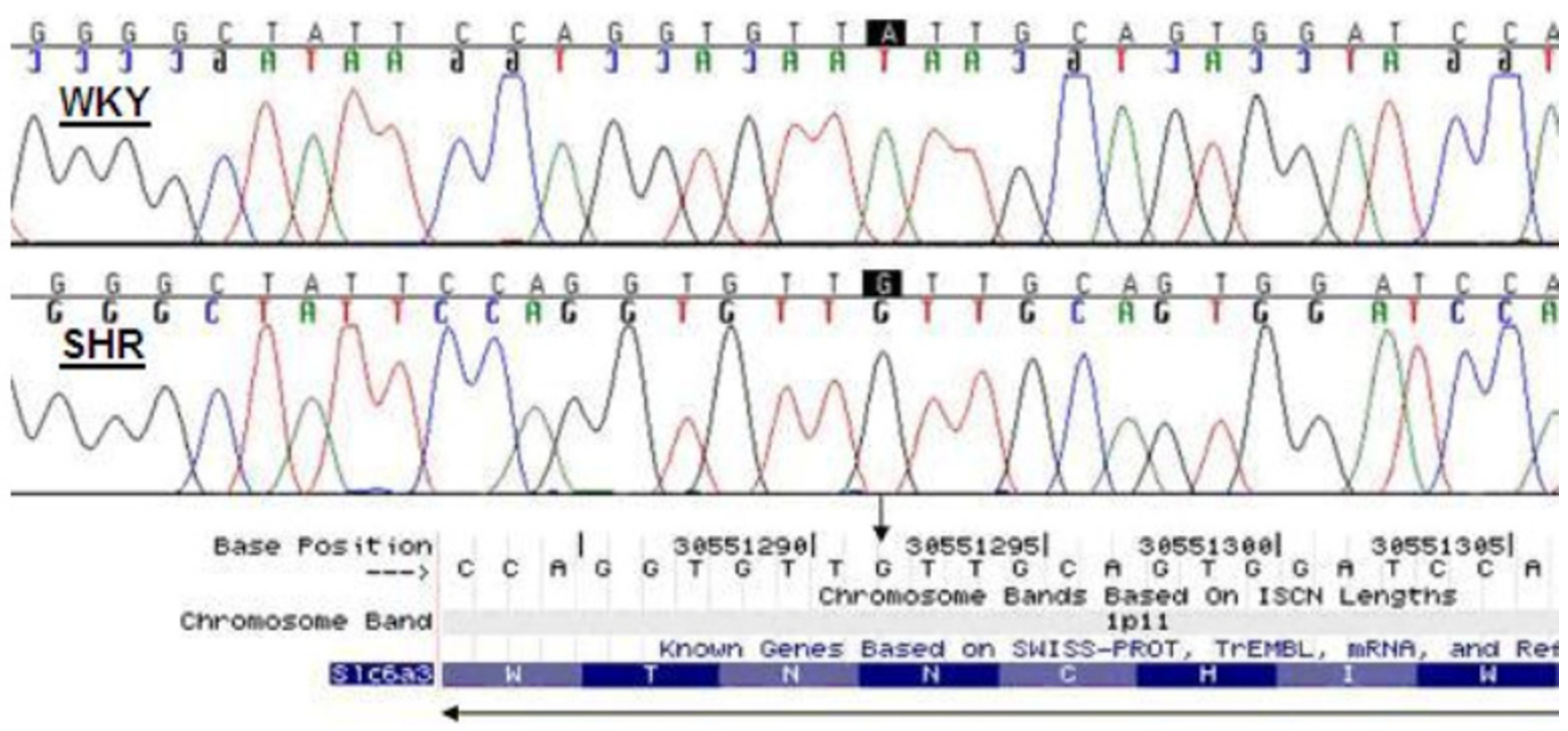

Figure 2

Single base polymorphism in exon 3 of Dat I: WKY (T) $\rightarrow$ SHR (C). The polymorphism is silent, and in both strains results in anasparagine amino-acid.

strains have been shown to exhibit elevated DAT expression in mesocortical projections $[16,17]$. It appears that excess DAT expression in the SHR may not be directly genetic in origin, but is in fact a response to excess mesocortical dopamine during early development resulting from hypofunctioning DAT protein that is presumably genetic $[2,16]$. The WKY strain also appears to have an unusual DAT profile compared to non-depressive control strains. Jiao et al report lower DAT density in the nucleus accumbens, amygdala, ventral tegmental area, and the reticular part of the substantia nigra of these animals, but higher expression in the hippocampus and hypothalamus [14].

It is interesting that these findings are partially mirrored in studies on human psychiatric patients. Whilst individuals with ADHD have been shown to exhibit increased DAT density in the brain $[18,19]$, depressive patients were found to have overall decreased levels of DAT [20]. Furthermore, genetic association studies suggests an association between a polymorphism in the human dopamine transporter gene (DAT1) and ADHD [21], although to date there is no evidence linking this polymorphism to the aetiology of depression.

\section{Conclusion}

In this study we have sequenced three dopaminergic genes in two inbred rat strains considered to be good models of human psychiatric illness. No between strain differences were observed in either the Drd2 or Drd4 genes, suggesting that neither gene is likely to mediate the behavioural differences observed between the WKY and SHR strains, although a number of polymorphisms common to both strains were detected in Drd4. In contrast, WKY/SHR differences were observed in the $3^{\text {rd }}$ exon of Dat1. Whilst these mutations do not result in direct amino-acid changes to the DAT protein, it is possible that they mediate some other process that explains the differences in DAT expression and function observed between the two strains. Future work should focus on further characterizing the genetic differences between these two strains, and investigating the functional consequences of the observed polymorphisms and how they relate to the putative depressive and hyperactive behaviours observed in the two strains.

\section{Competing interests}

The author(s) declare they have no competing interests.

\section{Authors' contributions}

JM carried out the molecular genetic work and drafted the manuscript. TS provided the animal tissue used in this 
study and participated in the overall study design. PA supervised the project and helped draft the manuscript. All authors read and approved the final manuscript.

\section{References}

I. Faraone SV, Perlis RH, Doyle AE, Smoller JW, Goralnick JJ, Holmgren MA, Sklar P: Molecular Genetics of Attention-Deficit/Hyperactivity Disorder. Biol Psychiatry 57( I I): | 3| 3-23. 6-I-2005

2. Russell VA, Sagvolden T, Johansen EB: Animal Models of Attention-Deficit Hyperactivity Disorder. Behav Brain Funct I:9. 7-I52005

3. Sagvolden T, Russell VA, Aase H, Johansen EB, Farshbaf M: Rodent Models of Attention-Deficit/Hyperactivity Disorder. Biol Psychiatry 57(I I): I239-47. 6-I-2005

4. Sagvolden T: Behavioral Validation of the Spontaneously Hypertensive Rat (SHR) As an Animal Model of AttentionDeficit/Hyperactivity Disorder (AD/HD). Neurosci Biobehav Rev 2000, 24(I):31-9.

5. Sagvolden T, Metzger MA, Schiorbeck HK, Rugland AL, Spinnangr I, Sagvolden G: The Spontaneously Hypertensive Rat (SHR) As an Animal Model of Childhood Hyperactivity (ADHD): Changed Reactivity to Reinforcers and to Psychomotor Stimulants. Behav Neural Biol 1992, 58(2): 103-12.

6. Russell V, de Villiers A, Sagvolden T, Lamm M, Taljaard J: Differences Between Electrically-, Ritalin- and D-Amphetamine-Stimulated Release of [3H]Dopamine From Brain Slices Suggest Impaired Vesicular Storage of Dopamine in an Animal Model of Attention-Deficit Hyperactivity Disorder. Behav Brain Res 1998, 94(I): 163-7|.

7. Russell VA: Dopamine Hypofunction Possibly Results From a Defect in Glutamate-Stimulated Release of Dopamine in the Nucleus Accumbens Shell of a Rat Model for Attention Deficit Hyperactivity Disorder - the Spontaneously Hypertensive Rat. Neurosci Biobehav Rev 2003, 27(7):67I-82.

8. Okamoto K, Aoki K: Development of a Strain of Spontaneously Hypertensive Rats. Jpn Circ J 1963, 27:282-93.

9. Huezo-Diaz P, Tandon K, Aitchison KJ: The Genetics of Depression and Related Traits. Curr Psychiatry Rep 2005, 7(2): I 7-24.

10. Dailly E, Chenu F, Renard CE, Bourin M: Dopamine, Depression and Antidepressants. Fundam Clin Pharmacol 2004, 18(6):60 I-7.

11. Lahmame A, del Arco C, Pazos A, Yritia M, Armario A: Are WistarKyoto Rats a Genetic Animal Model of Depression Resistant to Antidepressants? Eur J Pharmacol 337(2-3): I I5-23. 10-22- 1997

12. De La, Garza R, Mahoney JJ III: A Distinct Neurochemical Profile in WKY Rats at Baseline and in Response to Acute Stress: Implications for Animal Models of Anxiety and Depression. Brain Res I02 I(2):209-18. 9-24-2004

13. Will CC, Aird F, Redei EE: Selectively Bred Wistar-Kyoto Rats: an Animal Model of Depression and Hyper-Responsiveness to Antidepressants. Mol Psychiatry 2003, 8(I I):925-32.

14. Jiao X, Pare WP, Tejani-Butt S: Strain Differences in the Distribution of Dopamine Transporter Sites in Rat Brain. Prog Neuropsychopharmacol Biol Psychiatry 2003, 27(6):913-9.

15. Jeanpierre M: A Rapid Method for the Purification of DNA From Blood. Nucleic Acids Res I 5(22):96 I I. I I-25- 1987

16. Viggiano D, Grammatikopoulos G, Sadile AG: A Morphometric Evidence for a Hyperfunctioning Mesolimbic System in an Animal Model of ADHD. Behav Brain Res I30(I-2):181-9. 3-102002

17. Watanabe $Y$, Fujita M, Ito $Y$, Okada T, Kusuoka H, Nishimura T: Brain Dopamine Transporter in Spontaneously Hypertensive Rats. J Nucl Med 1997, 38(3):470-4.

18. Dougherty DD, Bonab AA, Spencer T], Rauch SL, Madras BK, Fischman AJ: Dopamine Transporter Density in Patients With Attention Deficit Hyperactivity Disorder. Lancet 354(9196):2132-3. 12-18-1999

19. Dresel S, Krause J, Krause KH, LaFougere C, Brinkbaumer K, Kung HF, Hahn K, Tatsch K. Attention Deficit Hyperactivity Disorder: Binding of [99mTc]TRODAT-I to the Dopamine Transporter Before and After Methylphenidate Treatment. Eur J Nucl Med 2000, 27(1 0):I5|8-24.

20. Meyer JH, Kruger S, Wilson AA, Christensen BK, Goulding VS, Schaffer A, Minifie C, Houle S, Hussey D, Kennedy SH: Lower Dopamine Transporter Binding Potential in Striatum During Depression. Neuroreport I2(18):4| 2|-5. |2-2|-200|
21. Madras BK, Miller GM, Fischman AJ: The Dopamine Transporter: Relevance to Attention Deficit Hyperactivity Disorder (ADHD). Behav Brain Res I30(I-2):57-63. 3-10-2002
Publish with Biomed Central and every scientist can read your work free of charge

"BioMed Central will be the most significant development for disseminating the results of biomedical research in our lifetime. "

Sir Paul Nurse, Cancer Research UK

Your research papers will be:

- available free of charge to the entire biomedical community

- peer reviewed and published immediately upon acceptance

- cited in PubMed and archived on PubMed Central

- yours - you keep the copyright
BioMedcentral 